J. Dairy Sci. 95:6145-6151

http://dx.doi.org/10.3168/jds.2012-5616

(C) American Dairy Science Association ${ }^{\circledR}, 2012$.

\title{
Genetic variation for infection status as determined by a specific antibody response against Mycobacterium avium subspecies paratuberculosis in milk of Dutch dairy goats
}

\author{
K. J. E. van Hulzen, ${ }^{11}$ A. P. Koets, ${ }^{*}$ M. Nielen, ${ }^{\star}$ J. Hoeboer, ${ }^{\star} \dagger$ J. A. M. van Arendonk,‡ \\ and H. C. M. Heuven§ \\ ${ }^{*}$ Department of Farm Animal Health, PO Box 80.151, and \\ †Department of Infectious Diseases and Immunology, PO Box 80.165, Utrecht University, 3508 TD Utrecht, the Netherlands \\ $\ddagger$ Animal Breeding and Genomics Centre, Wageningen University, PO Box 338, $6700 \mathrm{AH}$ Wageningen, the Netherlands \\ $\S$ Clinical Sciences of Companion Animals, Utrecht University, PO Box 80.163, 3508 TD Utrecht, the Netherlands
}

\section{ABSTRACT}

Classical control strategies based on management restrictions to reduce transmission, culling of infected goats, and vaccination have not been able to eradicate Johne's disease from infected herds. Selective breeding for less susceptibility to disease may be a useful additional tool to contribute to control of the disease. The aim of this study was to estimate genetic variation and heritability for infection status as determined by a specific antibody response against Mycobacterium avium subspecies paratuberculosis in milk of Dutch dairy goats. Milk samples from 950 goats were tested for antibodies specific to Johne's disease by ELISA on 5 consecutive test days, with a time interval of around 3 mo. Test results were coded as infected or not infected according to the instructions of the manufacturer. Heritability of infection status was estimated for 3 data sets to determine the effect of repeated sampling: only test results obtained on the first test day (first-test); the maximum test result of each animal obtained on 1 of the 5 test days (max-test); and all test results per animal, with a maximum of 5 consecutive samplings (alltest). Data sets first-test and max-test were analyzed with a sire model with fixed effects for year of birth and stage of lactation, and random effects for sire and error. For data set all-test, an additional permanent environment effect was included in the model. The estimated heritability on the underlying scale ranged from 0.12 in data set first-test, to 0.09 in data set max-test, to 0.07 in data set all-test.

Key words: Johne's disease, ELISA, repeated sampling, dairy goat

Received April 13, 2012

Accepted June 24, 2012

${ }^{1}$ Corresponding author: kimmvanhulzen@gmail.com

\section{INTRODUCTION}

Johne's disease, also known as paratuberculosis, is caused by oral uptake of Mycobacterium avium ssp. paratuberculosis (MAP) from the environment. Mycobacterium avium ssp. paratuberculosis causes granulomatous lesions in the distal part of the ileum in domestic and wild ruminants. In goats, ileal lesions limit sufficient nutrient uptake, leading to weight loss, a rough hair coat, and skin peeling. In the Dutch Holstein-Friesian dairy cattle population, prevalence of Johne's disease based on a positive ELISA test in milk was $2.4 \%$ in 2008 (van Hulzen et al., 2011). Prevalence of Johne's disease in dairy goat herds is unknown but suspected to be higher than in cattle herds, based on clinical and routine pathological observations.

Classical control strategies to eradicate MAP from infected goat farms are (1) management restrictions to reduce MAP transmission, (2) test and cull strategies to reduce the sources of infection, and (3) vaccination to decrease the susceptibility of the young stock. Management restrictions to reduce MAP transmission focus mainly on avoiding contact of young, susceptible stock with infected animals, such as separation of kids from dams immediately after birth. For test and cull strategies to reduce the sources of infection, ELISA are most commonly used because of their simplicity and low cost. In cows, ELISA are considered to be highly specific, but of low sensitivity (Whitlock et al., 2000). This is because the humoral response, as measured by ELISA, is associated with later stages of disease. Moreover, when animals reach the advanced stage of infection in which they show a humoral response, the response may be intermittent. Antibody detection by ELISA, even in animals in advanced stages of disease, may, therefore, be unreliable. Repeated testing of animals over a longer time period can be applied to increase the probability of antibody detec- 
tion in infected animals and to reduce the sources of infection. Vaccination is the principal control strategy for caprine Johne's disease in the Netherlands. Nevertheless, despite the effectiveness of the vaccine in reducing the number of clinical cases on the farm, continuous shedding of MAP is suspected and may be a source for new infections. In Norway, a vaccination program was initiated in 1967 after several years of unsuccessful efforts to eradicate paratuberculosis in goats. The efficacy of the vaccine was judged mainly by postmortem examination of vaccinated and unvaccinated goats in the period 1967 to 1982 and results showed that MAP vaccination offers a high degree of protection to infection (Saxegaard and Fodstad, 1985). In Australia, MAP vaccination of Merino flocks was usually effective in reducing prevalence of shedding, but the response to vaccination in the different flocks was variable (Dhand et al., 2012). Bastida and Juste (2011) summarized results of vaccination experiments and showed that MAP vaccination performed well in reducing production, epidemiological, and pathogenetic effects in cows, sheep and goats. Although these classical control strategies are able to reduce the rate of infection considerably, eradication of MAP has been shown to be difficult and additional approaches to address Johne's disease are needed. One additional approach is selective breeding for animals less susceptible to Johne's disease.

In cows, the heritability of susceptibility to Johne's disease ranged from 0.03 to 0.23 (Koets et al., 2000; Mortensen et al., 2004; Gonda et al., 2006; Hinger et al., 2007; Attalla et al., 2010; van Hulzen et al., 2011; Küpper et al., 2012). In goats, Singh et al. (2009) found differences in susceptibility to Johne's disease between 5 native breeds in 2 agro-climates in India. Differences in susceptibility were determined by examination of BW, morbidity, and mortality due to Johne's disease, fecal shedding of MAP, and gross and microscopic lesions in target tissues. In the study of Singh et al. (2012), goats were classified as resistant and susceptible based on clinical signs, microscopic examination, fecal culture, ELISA, and diagnostic PCR. Analysis of the polymorphism in the exon- 2 of the caprine major histocompatibility complex class II $D R B$ gene in the susceptible and resistant goats showed association with susceptibility to Johne's disease. However, quantification of the genetic variation within a dairy goat population, by using phenotypes for susceptibility to Johne's disease and pedigree information, is lacking. The objective of this study was to estimate genetic variation and heritability for infection status, as determined by a specific antibody response against MAP in milk of Dutch dairy goats.

\section{MATERIALS AND METHODS}

\section{Animals}

Nine hundred fifty goats originating from 1 nonvaccinated herd with endemic MAP infection were included in the study. Goats were selected based on age (at least $2 \mathrm{yr}$ old at the time of selection) and known sire. Selected goats originated from 56 sires and 847 dams, and were mainly purebreds of the Dutch White milking goat breed, which is closely related to the Saanen breed. Pedigrees and milk production records of the animals were provided by ELDA ICT \& Services BV (Rijen, the Netherlands). For each goat, sire pedigree information of at least 3 generations was available.

\section{Samples}

From May 2009 to June 2010, milk samples were collected during 5 consecutive samplings with a time interval of around 3 mo (Figure 1). During the study period, goats were culled based on positive test results in a MAP ELISA, illness, or low milk production, which decreased the number of tested goats at later test days. The number of tested goats on test $\mathrm{d} 2$ is less compared with test d 3 due to technical problems with electronic animal identification.

\section{Antibody Detection}

All samples were tested using a commercially available ELISA kit (ELISA paratuberculosis antibody screening; Institut Pourquier, Montpellier, France) according to the instructions of the manufacturer. Results were expressed as percentage of the sample to positive ratio $(S / P)$, calculated as $100 \times$ [the optical density $($ OD) value of the sample - the OD value of the negative control]/[the OD value of the positive control - the OD value of the negative control], and coded binary with a cut-off of 40, as recommended by the manufacturer.

Until now, no official validation for the use of ELISA on milk samples of goats is available. In this study, the use of ELISA in milk was preferred because of logistic advantages that allow testing of large number of goats for infection with Johne's disease. To be able to assess the correlation between ELISA results in serum and in milk, 62 paired serum and milk samples from goats at risk for paratuberculosis were pretested.

\section{Effect of Repeated Measures}

To quantify the effect of repeated measures, genetic parameters were estimated on 3 subsets of the col- 


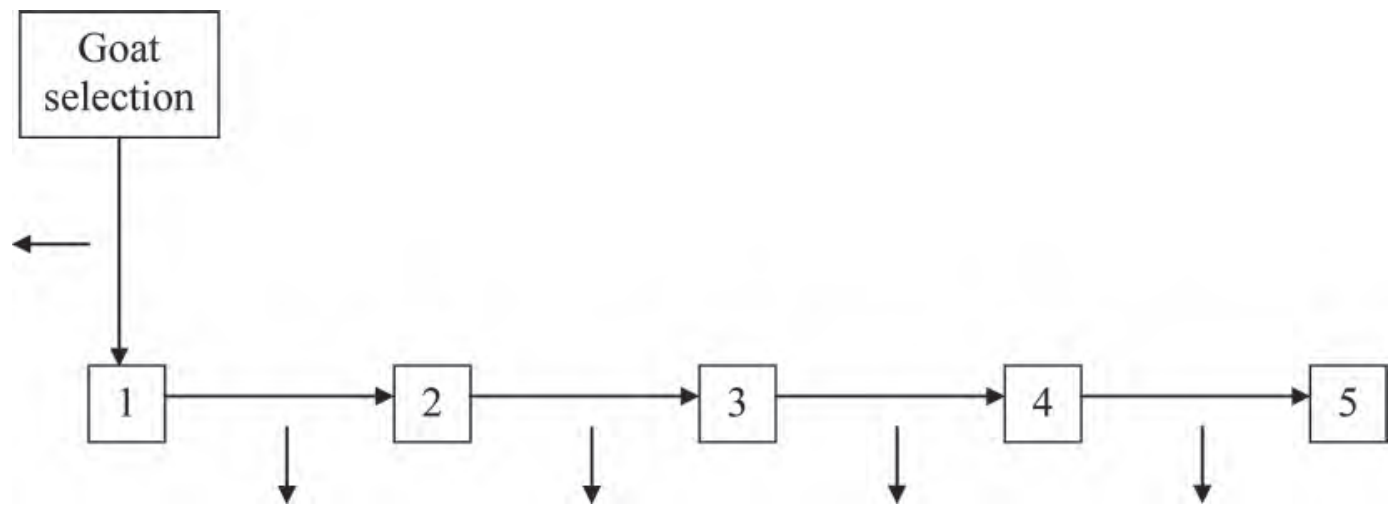

Figure 1. Sampling flowchart: at the start of the study, 950 goats were selected based on age and known sire. Numbers 1 to 5 represent single test days, with a time interval of around 3 mo. Culling during the research period (indicated by left-pointing arrow and downward-pointing arrows) was due to (1) selection of animals a few months before the start of data collection; (2) decision of the farmer, based on low production or disease; or (3) decision of the farmer, based on positive ELISA results.

lected data: test results obtained on the first test day (first-test); the maximum test result of each animal obtained on 1 of its test days (max-test); and all test results per animal, with a maximum of 5 consecutive samplings (all-test). All data sets were edited to meet the minimum requirement of 5 daughters per sire. In total, 950 animals were included in data set max-test and all-test and of these, 878 animals were included in data set first-test.

\section{Statistical Analysis}

Variance components for the genetic effect for data sets first-test and max-test were estimated using a sire model with a logit link in ASReml (Gilmour et al., 2009):

$$
Y_{i j k}=\mu+Y B_{i}+L S_{j}+\operatorname{sire}_{k}+e_{i j k}
$$

and for data set all-test, a repeated measures sire model with a logit link was used:

$$
Y_{i j k l}=\mu+Y B_{i}+L S_{j}+\text { sire }_{k}+p e_{l}+e_{i j k l},
$$

where $Y_{i j k(l)}$ is the binary ELISA test result of the individual goat, $\mu$ is the general mean, $Y B_{i}$ is the fixed effect of the $i$ th year of birth $(i=2005,2006,2007$, or $\leq 2004), L S_{j}$ is the fixed effect of the $j$ th stage of lactation $(j=0-50,51-100,100-200,201-450$, or $\geq 451 \mathrm{~d})$, sire $_{k}$ is the random genetic effect of the $k$ th sire, $p e_{l}$ is the random effect of the $l$ th animal (permanent environment), and $e_{i j k(l)}$ is the random residual component. The following distributional assumptions were made for the random effects:

$$
\operatorname{sire} \sim N\left(0, \mathbf{A} \sigma_{s}^{2}\right)
$$

Table 1. Variance components and heritability (SE of heritability in parentheses) for infection status as determined by a Mycobacterium avium subspecies paratuberculosis-specific antibody response in milk of Dutch dairy goats for data set first-test (test results obtained on test d 1), data set max-test (the maximum test result of each animal obtained on 1 of its 5 test days), and data set all-test (all test results per animal, with a maximum of 5 consecutive samplings)

\begin{tabular}{lcccccc}
\hline & \multicolumn{5}{c}{ Variance component $^{1}$} \\
\cline { 2 - 6 } Data set & $\sigma_{s}^{2}$ & $\sigma_{a}^{2}$ & $\sigma_{p e}^{2}$ & $\sigma_{e}^{2}$ & $\sigma_{p}^{2}$ & $\mathrm{~h}^{2}$ \\
\hline First-test $^{2}$ & 0.10 & 0.40 & - & 3.29 & 3.39 & $0.12(0.23)$ \\
Max-test $^{2}$ & 0.08 & 0.31 & - & 3.29 & 3.37 & $0.09(0.13)$ \\
All-test & 0.09 & 0.36 & 2.17 & 3.29 & 5.55 & $0.07(0.09)$ \\
\hline${ }^{1} \sigma_{s}^{2}=$ sire variance; $^{2} \sigma_{a}^{2}=$ additive genetic variation; $\sigma_{p e}^{2}=$ permanent environmental variance; $\sigma_{e}^{2}=$ residual \\
variance; $\sigma_{p}^{2}=$ phenotypic variation. \\
${ }^{2}$ Estimated heritability differed significantly from zero $(P<0.05)$.
\end{tabular}


and

$$
\mathbf{p e} \sim N\left(0, \mathbf{I} \sigma_{p e}^{2}\right)
$$

only for model 2 , where sire is the vector included in the sire additive genetic effect, pe is the vector included in the animal effect (permanent environment effect), $\mathbf{A}$ is the sire relationship matrix, $\sigma_{s}^{2}$ represents the sire variance, $\mathbf{I}$ is the identity matrix, and $\sigma_{p e}^{2}$ represents the permanent environmental variance. Variance components were used to estimate the heritability $\left(h^{2}\right)$ on the underlying scale, which was defined as follows: $h^{2}=\sigma_{a}^{2} / \sigma_{p}^{2}$, where $\sigma_{a}^{2}$ is the additive genetic variation, defined as $4 \times \sigma_{s}^{2}$; and $\sigma_{p}^{2}$ is the phenotypic variation, defined as $\sigma_{s}^{2}+\sigma_{e}^{2}$ for model 1 and as $\sigma_{s}^{2}+\sigma_{p e}^{2}+\sigma_{e}^{2}$ for model 2, where $\sigma_{e}^{2}$ represents the residual variance, which was fixed at $\frac{\pi^{2}}{3}$.

To test the hypothesis that the heritability is significantly different from zero, a likelihood ratio test (LRT) was performed as twice the difference in log-likelihoods between the full model (model 1 and 2) and the reduced model without a random sire effect. For a single variance component, the theoretical asymptotic distribution of the LRT is a mixture of $\chi^{2}$ variates, where the mixing probabilities are 0.5 , one with 0 degrees of freedom and the other with 1 degree of freedom that has a $5 \%$ critical value of 2.71 (Gilmour et al., 2009).

\section{Model Comparison}

To maximize genetic response for the trait of interest, superior breeding stock for the trait of interest must be accurately identified and selected. Model comparison was used to assess the consistency of results provided by model 1 , including first-test data; model 1 , including the max-test data; and model 2 including all-test data. Estimated breeding values of 56 sires were compared using Spearman rank correlation coefficients in R software (package 2.90; http://www.r-project.org).

\section{RESULTS}

\section{Correlation Between ELISA in Serum Versus Milk}

To assess the correlation between ELISA results in serum and in milk, 62 paired serum and milk samples from goats at risk for paratuberculosis were pretested. Out of 62 goats, 54 goats tested negative in serum and milk and 6 goats tested positive in serum and milk. One goat tested negative in serum and positive in milk and 1 goat tested positive in serum and negative in milk.

\section{Prevalence}

Prevalence of infection in the selected group, as measured by a positive ELISA in milk, was $6 \%$ on the first test day (51 out of 878 goats tested positive). Although goats were culled based on positive test results in the ELISA during the study period, test prevalence remained quite stable: $4 \%$ on test d 2 (25 out of 668 ), $6 \%$ on test d 3 (43 out of 712 ), $8 \%$ on test d 4 (47 out of 621 ), and $2 \%$ on test d 5 (10 out of 552). Prevalence of infection in the max-test data set, containing the maximum test result of each animal obtained on 1 out of 5 test days was 0.13 . Prevalence of infection in the all-test data set was 0.05 .

\section{Genetic Variation and Heritability}

Genetic variation and heritability were estimated for infection status as measured by MAP-specific antibody response in Dutch dairy goats using 3 sets of data (Table 1). Additive genetic variance was largest in data set first-test, followed by data set all-test and data set max-test. The estimated heritability on the underlying scale ranged from 0.12 in data set first-test, to 0.09 in data set max-test, to 0.07 in data set all-test. Estimated heritability differed significantly from zero $(P<0.05)$ for first-test and max-test only.

\section{Model Comparison}

Spearman rank correlation coefficients were calculated between breeding values estimated from 3 different combinations of input data set and model and are shown in Table 2. The largest correlation was between max-test and all-test (0.90). Correlations between firsttest and max-test, and first-test and all-test were lower (0.61 and 0.68, respectively).

\section{DISCUSSION}

This study estimated genetic variation and heritability for infection status as determined by a specific antibody response against MAP in milk in 1 dairy goat herd in the Netherlands to investigate if genetic selection for less susceptibility to Johne's disease can contribute to control of disease. Pretesting of paired serum and milk samples from 62 goats at risk for paratuberculosis showed a high correlation in infection status as determined by an ELISA. Another study estimated the concordance between paired serum and milk of dairy goats at 4 stages of lactation and showed only a significant difference between the proportion of positive serum samples and milk in late lactation. In late lactation, milk testing may outperform serological 
Table 2. Spearman rank correlation coefficients between 56 sire breeding values estimated from model 1, including test results obtained on the first test day (first-test); model 1, including the maximum test result of each animal obtained on 1 of its test days (max-test); and model 2, including all test results per animal with a maximum of 5 consecutive samplings (all-test)

\begin{tabular}{lcccc}
\hline & \multicolumn{2}{c}{ Model 1} & & Model 2 \\
\cline { 2 - 3 } Data set & First-test & Max-test & & All-test \\
\hline First-test & 1 & 0.61 & & 0.68 \\
Max-test & - & 1 & & 0.90 \\
All-test & - & - & \\
\hline
\end{tabular}

testing (Aggelidou et al., 2012). Both studies show high correlation between ELISA results in serum and milk and, therefore, an ELISA in milk was selected.

The sensitivity of the ELISA in milk of goats is unknown but expected to be low. Because the humoral response, as measured by ELISA, is associated with later stages of disease, the probability of antibody detection in an infected goat increases with age. To increase the probability of detecting the humoral response, only goats of at least $2 \mathrm{yr}$ of age were included in the study and repeated testing was applied up to 5 times, with an interval of around 3 mo. Repeated testing would allow the calculation of repeatability. However, (technical) calculation of repeatability does not provide any biological information for this data. This is mainly due to the low sensitivity of the test combined with the potential change in antibody titer of infected animals over the 1-yr period.

Goats with a MAP-specific antibody response above a defined threshold were considered to be infected. Because the process of infection is said to take place at an early age and antibody detection develops in later stages of disease, only goats of at least $2 \mathrm{yr}$ of age were included in this study to increase the probability of detecting infection. The factors age, stage of lactation, parity, and number of kiddings during the study period were considered for correction in the models by backward elimination. Age and stage of lactation appeared to have a significant effect $(P<0.05)$ on infection status as determined by a MAP-specific antibody response in all data sets.

For cows in the Netherlands, prevalence of a positive ELISA test in milk was $2.4 \%$ on the animal level and $46.7 \%$ on the herd level in 2008 (van Hulzen et al., 2011). Although the prevalence of infection on the goat level was expected to be higher than in cows, based on clinical and routine pathological observations, prevalence was measured in a selected group within 1 herd. The intensive use of vaccination in the general Dutch goat population hampers the estimation of the true prevalence, both on the animal level and herd level.
According to the test prevalence in the different data sets, additive genetic variation was expected to be comparable in first-test and all-test data sets, but additive genetic variation was expected to be highest in the max-test data set. No confounding occurred between the genetic effect and year of birth or stage of lactation. The $F$-value for year of birth was highest in the first-test data set and estimates for the different year of birth categories showed low values for the youngest goats included in the study (year of birth 2007). In max-test and all-test data sets, the $F$-values for year of birth were lower and estimates for the different year of birth categories were much more comparable. This indicates that in the first-test data set, the probability of antibody detection in the youngest infected goats was much lower, even though goats were selected based on age to exclude goats in early stages of disease without a detectable humoral response. Repeated testing over a 1-yr period increased the probability of antibody detection of the goats of at least $2 \mathrm{yr}$ of age at the start of the study.

Standard errors of heritability estimates were large (0.09 to 0.23 ). The standard error of the heritability is useful as an indicator of the precision of the heritability estimate but it is better to use an LRT as a formal test of a component. In contrast to first-test and max-test data, the estimated heritability was not significantly different from zero $(P<0.05)$ according to the LRT for all-test data. Confounding between the sire effect and the permanent environment effect was observed and resulted in a nonsignificant heritability for all-test data.

Low Spearman rank correlation coefficients between first-test and max-test data, and first-test and alltest data indicate that changes in EBV ranking are to be expected when comparing selection candidates originating from analysis of the first-test data set with selection candidates originating from analysis using the repeated measures. This is most likely due to an improvement in disease phenotype when using repeated measures over time, combined with age clustered sires. As mentioned earlier, testing at the first time point (first-test) provides positive tests mainly for older goats that are more likely to be in advanced stages of disease. As a result, differences between sires in genetic capacity are observed, but in reality, younger sires do not have test-positive progeny yet and are, therefore, classified as resistant. Data showed that susceptible sires with only 2-yr-old progeny at the start of the study were classified as resistant in the analysis of first-test data and classified as susceptible in the analysis of max-test and all-test data.

The high Spearman rank correlation coefficient between the max-test and all-test data sets indicates that sires were ranked almost the same when using 
the maximum test result of each animal obtained on 1 of the 5 test days or all test results per animal, with a maximum of 5 consecutive samplings. However, in our opinion, estimation of genetic variation and heritability in this study should preferably be based on the maximum ELISA result of each animal obtained by multiple testing over a longer (1-yr) time period to reduce the number of false-negative results included in the analysis.

In this study, genetic variation for infection status as determined by a MAP-specific antibody response was quantified in Dutch dairy goats. However, knowledge of genes contributing to genetic variance of susceptibility to caprine Johne's disease is still very limited. In the study of Singh et al. (2012), analysis of the polymorphism in the exon-2 of the major histocompatibility complex class II $D R B$ gene showed association with susceptibility to caprine Johne's disease. As Johne's disease progression contains different stages, a variety of immunological mechanisms may be used to combat infection, which suggests involvement of a large number of genes in susceptibility to Johne's disease, each having a small effect.

Potential for genetic improvement of susceptibility to Johne's disease has been shown; however, genetic variation and heritability were estimated on herd level and displayed on an underlying scale. Among other factors, goats included in this study were selected based on known sire. In the Netherlands, pedigree registration is mainly applied when using AI and, as a result, numerous selected goats originated from billy goats that are used for AI on a national scale. Also, in terms of management factors to reduce transmission of Johne's disease (separate rearing of kids and culling of diseased animals), the farm is representative for Dutch dairy farms. Even though the existence of genetic variation on a national scale is still unknown and difficult to estimate because pedigree registration is not common and vaccination hampers diagnosis, genetic variation for susceptibility to caprine Johne's disease on a national scale is expected to be comparable to estimates in this study. Genetic variation and heritability in Dutch dairy cows were in the range of those in the present study and heritability estimates varied from 0.04 to 0.10 , depending on the herd test prevalence (van Hulzen et al., 2011). Further research should include the effect of selection for goats less susceptible to Johne's disease on other (production) traits.

\section{CONCLUSIONS}

Differences were observed between goats in their susceptibility to infection with Johne's disease as de- termined by a specific antibody response against MAP. For this reason, it seems feasible to breed for animals less susceptible to disease to contribute to a more effective control of Johne's disease. Estimated heritability varied between 0.07 and 0.12 in different subsets of data. Spearman rank correlation coefficients showed that changes in EBV ranking are to be expected when comparing selection candidates originating from the different subsets of data. This difference is most likely due to an improvement in disease phenotype when using repeated measures over a longer time.

\section{ACKNOWLEDGMENTS}

The authors thank the farmer for participation in the study; ELDA ICT Services BV (Rijen, the Netherlands) for supplied pedigree information, milk production records, and support where needed; and Marina Bouman (University of Utrecht, Utrecht, the Netherlands) for her assistance and guidance in the laboratory. Many thanks to Judith Dijcker, Wiebren Santema, Gerrit Koop, Susanne Eisenberg, Marina Bouman, Boris Schmid, Lindert Benedictus, Sanne Slot, Manouk Vrieling, Thijs Wessels, and Gerrit Oosterhuis (all of University of Utrecht) for their excellent assistance during the sampling days.

\section{REFERENCES}

Aggelidou, E., P. Kostoulas, and L. Leontides. 2012. Concordance between Mycobacterium avium subsp. paratuberculosis ELISA results in paired sera and milk of dairy goats. Page 357 in Proc. 11th Int. Colloq. Paratuberculosis, University of Sydney, Australia. Int. Assoc. Paratuberculosis, Sydney, Australia.

Attalla, S. A., A. J. Seykora, J. B. Cole, and B. J. Heins. 2010. Genetic parameters of milk ELISA scores for Johne's disease. J. Dairy Sci. 93:1729-1735.

Bastida, F., and R. A. Juste. 2011. Paratuberculosis control: A review with a focus on vaccination. J. Immune Based Ther. Vaccines 9:8.

Dhand, N. K., J. Eppleston, and P. A. Windsor. 2012. Evaluation of the effectiveness of vaccination for reducing ovine Johne's disease prevalence in sheep flocks in Australia. Page 333 in Proc. 11th Int. Colloq. Paratuberculosis, University of Sydney, Australia. Australia. Int. Assoc. Paratuberculosis, Sydney, Australia.

Gilmour, A. R., B. J. Gogel, B. R. Cullis, and R. Thompson. 2009. ASReml User Guide. Release 3.0. VSN International Ltd., Hemel Hempstead, UK.

Gonda, M. G., Y. M. Chang, G. E. Shook, M. T. Collins, and B. W. Kirkpatrick. 2006. Genetic variation of Mycobacterium avium ssp. paratuberculosis infection in US Holsteins. J. Dairy Sci. 89:18041812.

Hinger, M., H. Brandt, S. Horner, and G. Erhardt. 2007. Short communication: Association analysis of microsatellites and Mycobacterium avium subspecies paratuberculosis antibody response in German Holsteins. J. Dairy Sci. 90:1957-1961.

Koets, A. P., G. Adugna, L. L. G. Janss, H. J. van Weering, C. H. J. Kalis, G. H. Wentink, V. P. M. G. Rutten, and Y. H. Schukken. 2000. Genetic variation of susceptibility to Mycobacterium avium subsp. paratuberculosis infection in dairy cattle. J. Dairy Sci. $83: 2702-2708$ 
Küpper, J., H. Brandt, K. Donat, and G. Erhardt. 2012. Heritability estimates for Mycobacterium avium subspecies paratuberculosis status of German Holstein cows tested by fecal culture. J. Dairy Sci. 95:2734-2739.

Mortensen, H., S. S. Nielsen, and P. Berg. 2004. Genetic variation and heritability of the antibody response to Mycobacterium avium subspecies paratuberculosis in Danish Holstein cows. J. Dairy Sci. $87: 2108-2113$.

Saxegaard, F., and F. H. Fodstad. 1985. Control of paratuberculosis (Johne's disease) in goats by vaccination. Vet. Rec. 116:439-441.

Singh, P. K., S. V. Singh, A. V. Singh, and J. S. Sohal. 2009. Variability in susceptibility of different Indian goat breeds with respect to natural and experimental infection of Mycobacterium avium subspecies paratuberculosis. Indian J. Small Rumin. 15:35-43.
Singh, P. K., S. V. Singh, M. K. Singh, V. K. Saxena, P. Horin, A. V. Singh, and J. S. Sohal. 2012. Effect of genetic variation in the $M H C$ Class II $D R B$ region on resistance and susceptibility to Johne's disease in endangered Indian Jamunapari goats. Int. J. Immunogenet. 39:314-320. http://dx.doi.org/10.1111/j.1744313X.2012.01092.x

van Hulzen, K. J. E., M. Nielen, A. P. Koets, G. de Jong, J. A. M. van Arendonk, and H. C. M. Heuven. 2011. Effect of herd prevalence on heritability estimates of antibody response to Mycobacterium avium subspecies paratuberculosis. J. Dairy Sci. 94:992-997.

Whitlock, R. H., S. J. Wells, R. W. Sweeney, and J. Van Tiem. 2000. ELISA and fecal culture for paratuberculosis (Johne's disease): Sensitivity and specificity of each method. Vet. Microbiol. $77: 387-398$. 